\title{
Report of Meeting
}

\section{The Sixteenth Debrecen-Katowice Winter Seminar Hernádvécse (Hungary), January 27-30, 2016}

The Sixteenth Debrecen-Katowice Winter Seminar on Functional Equations and Inequalities was held in Hotel Vécsecity, Hernádvécse, Hungary, from January 27 to 30, 2016. It was organized by the Department of Analysis of the Institute of Mathematics of the University of Debrecen.

The Winter Seminar was supported by the following organizations:

- Institute of Mathematics, University of Debrecen;

- Hungarian Scientific Research Fund Grant OTKA K-111651.

27 participants came from two countries, 12 from the University of Silesia in Katowice (Poland), 1 from the Pedagogical University in Kraków (Poland), 11 from the University of Debrecen (Hungary), and 3 from the University of Miskolc (Hungary).

Professor Zsolt Páles opened the Seminar and welcomed the participants to Hernádvécse.

The scientific talks presented at the Seminar focused on the following topics: equations in a single variable and in several variables, iterative equations, equations on algebraic structures, preserver problems, equations on restricted domains, regularity properties of the solutions of certain functional equations, functional inequalities, Hyers-Ulam stability, functional equations and inequalities involving mean values, generalized convexity, convergence of Fourier series. Interesting discussions were generated by the talks.

There were profitable Problem Sessions.

The social program included a visit to Vizsoly, where the oldest complete Bible in Hungarian language is exhibited, and a Festive Dinner. Furthermore, the owner of the hotel guided the participants in the fascinating, city-like 
interior of the castle hotel, and he also explained some features of the reconstruction of the old castle. At the end of this guided tour, a ride in a horse-drawn carriage was organized around the castle park.

The closing address was given by Professor Roman Ger. His invitation to the Seventeenth Katowice-Debrecen Winter Seminar on Functional Equations and Inequalities from February 1 to 4, 2017 in Poland was gratefully accepted.

Summaries of the talks in alphabetic order of the authors follow in section 1, problems and remarks in chronological order in section 2, and the list of participants in the final section.

\section{Abstracts of talks}

Roman BAdora: On a Shtern generalization of a Ger-̌̌emrl theorem

In Exponential stability of quasihomomorphisms into Banach algebras and a Ger-Šemrl theorem (Russ. J. Math. Phys. 22 (2015), 141-142) A. I. Shtern proposed the following

(S) Let $(S ;+)$ be a semigroup, and let $A$ be a complex Banach algebra. Assume that the mapping $f: S \rightarrow A$ is such that

(a) the transformation

$$
S^{2} \ni(x, y) \longmapsto f(x+y)-f(x) f(y) \in A
$$

is norm bounded,

(b) every nonzero $S$-orbit

$$
O_{S}(b)=\{f(x) b: x \in S\}, \quad b \in A,
$$

in $A$ is unbounded.

Then $f$ is exponential, i.e., $f(x+y)=f(x) f(y)$ for any $x, y \in S$.

as a generalization of the Ger-Šemrl theorem (see Ger R., Šemrl P., The stability of the exponential equation, Proc. Amer. Math. Soc. 124 (1996), no. 3, 779-787).

Theorem (Ger, Šemrl). Let $(S ;+)$ be a semigroup, and let $A$ be a commutative semisimple complex Banach algebra. Assume that the mapping $f$ : $S \rightarrow A$ is such that 
(a) the transformation

$$
S^{2} \ni(x, y) \longmapsto f(x+y)-f(x) f(y) \in A
$$

is norm bounded,

(b) for every nonzero linear multiplicative functional $\varphi$ on $A$, the $\operatorname{set}(\varphi \circ f)(S)$ is unbounded.

Then $f$ is exponential.

In the talk we give an example which shows that $(S)$ is not true and we present a generalization the Shtern type of the Ger-ŠSemrl theorem.

Zoltán Boros: A dichotomy property for the graphs of monomials (Joint work with José Maria Almira)

Let $m$ be a positive integer. We call $f: \mathbb{R} \rightarrow \mathbb{R}$ monomial of degree $m$ if

$$
\Delta_{y}^{m} f(x)=m ! f(y) \quad(x, y \in \mathbb{R})
$$

We say that $f$ is a monomial function if $f$ is a monomial of degree $m$ for a positive integer $m$. In this talk, we present a more or less straightforward proof of the following dichotomy result.

TheOREm. Let $f: \mathbb{R} \rightarrow \mathbb{R}$ be a monomial function. Then its graph

$$
G(f)=\{(x, f(x)): x \in \mathbb{R}\}
$$

is either connected or totally disconnected.

PÁL BuRAI: Generalized Wright-convex functions (Joint work with Judit Makó)

The aim of this talk is the investigation of generalized Wright-convex functions.

\section{REFERENCES}

[1] Bessenyei M., Páles Zs., Characterization of higher-order monotonicity via integral inequalities, Proc. Roy. Soc. Edinburgh Sect. A 140 (2010), no. 4, 723-736.

[2] Bernstein F., Doetsch G., Zur Theorie der konvexen Funktionen, Math. Ann. 76 (1915), $514-526$.

[3] Kominek Z., A continuity result on t-Wright convex functions, Publ. Mat. 63 (2003), 213-219.

[4] Maksa Gy., Nikodem K., Páles Zs., Results on t-Wright convexity, C. R. Math. Rep. Acad. Sci. Canada 13 (1991), no. 6, 274-278. 
[5] Ng C.T., Functions generating Schur-convex sums, in: General inequalities, 5 (Oberwolfach, 1986), Internat. Schriftenreihe Numer. Math. vol. 80, Birkhäuser, Basel, 1987, pp. $433-438$.

[6] Olbryś A., Some conditions implying the continuity of t-Wright convex functions, Publ. Math. Debrecen 68 (2006), no. 3-4, 401-418.

[7] Olbryś A., Representation theorems for $t$-Wright convexity, J. Math. Anal. Appl. 384 (2011), no. 2, 273-283.

[8] Olbryś A., On some inequalities equivalent to the Wright-convexity, J. Math. Inequal. 9 (2015), no. 2, 449-461.

[9] Wright E.M., An inequality for convex functions, Amer. Math. Monthly 61 (1954), $620-622$.

\section{Szymon Draga: Means of iterates}

Let $n$ be a positive integer and $I \subset \mathbb{R}$ be an interval. Using results from [1] it is easy to determine all continuous functions $f: I \rightarrow I$ satisfying

$$
\frac{f(x)+\ldots+f^{n}(x)}{n}=x .
$$

In view of this observation it is natural to ask about continuous functions $f: I \rightarrow I$ satisfying

$$
f^{n}(x)=\frac{x+f(x)+\ldots+f^{n-1}(x)}{n} .
$$

During the talk we solve the above functional equation as well as we determine all continuous functions whose $n$th iterate is a power mean or the geometric mean of iterates of lower order.

\section{REFERENCE}

[1] Draga S., Morawiec J., Reducing the polynomial-like iterative equations order and a generalized Zoltán Boros problem, Aequationes Math. (2016), DOI 10.1007/s00010-0160420-4.

WŁodzIMIERZ FECHNER: Systems of inequalities characterizing ring homomorphisms (Joint work with Andrzej Olbryś)

Let $(P, \leq)$ and $(R, \leq)$ be partially ordered rings such that:

(A1) every nonnegative element of $P$ is a square:

$$
\forall_{f \in P, f \geq 0} \exists_{g \in P}\left[f=g^{2}\right],
$$

(A2) every square in $R$ is nonnegative:

$$
\forall_{g \in R}\left[g^{2} \geq 0\right] .
$$


Next, let $T: P \rightarrow R$ and $U: P \rightarrow R$ be two (unknown) mappings. We present results on various systems of functional inequalities for $T$ and $U$. In particular, we show that if $T$ and $U$ satisfy the system:

$$
\left\{\begin{aligned}
T(f+g) & \geq T(f)+T(g) \\
U(f \cdot g) & \geq U(f) \cdot U(g)
\end{aligned}\right.
$$

and $U \leq T$, then $U=T$ and this mapping is a ring homomorphism. Moreover, the same assertion is true if $T$ and $U$ satisfy the system:

$$
\left\{\begin{aligned}
T(f+g) & \geq U(f)+T(g) \\
U(f \cdot g) & \geq T(f) \cdot T(g)
\end{aligned}\right.
$$

György GáT: Summability of one and two dimensional Walsh-Fourier series, convergence and divergence, some recent results

Let $x$ be an element of the unit interval $I:=[0,1)$. The $\mathbb{N} \ni n$th Walsh function is

$$
\omega_{n}(x):=(-1)^{\sum_{k=0}^{\infty} n_{k} x_{k}}, \quad n=\sum_{k=0}^{\infty} n_{k} 2^{k}, x=\sum_{k=0}^{\infty} \frac{x_{k}}{2^{k+1}} .
$$

The $n$th Walsh-Fourier coefficient, the $n$th partial sum of the Fourier series, the $n$th $(C, 1)$ mean of $f \in L^{1}(I)$ :

$$
\hat{f}(n):=\int_{I} f(x) \omega_{n}(x) d x, \quad S_{n} f:=\sum_{k=0}^{n-1} \hat{f}(k) \omega_{k}, \quad \sigma_{n} f:=\frac{1}{n} \sum_{k=0}^{n-1} S_{k} f .
$$

It is of main interest how to reconstruct a function from the partial sums of its Walsh-Fourier series. Fine proved [1] that for each integrable function we have the almost everywhere convergence of Fejér means $\sigma_{n} f \rightarrow f$. In the talk we give a brief résumé of the recent results (see e.g. [2]) with respect to summability of one and two-dimensional Walsh-Fourier series. Among others, we investigate properties of various summability methods with respect to some orthonormal systems (Walsh-Paley, trigonometric) by the help of Haar-like functions [3].

\section{REFERENCES}

[1] Fine N.J., Cesàro summability of Walsh-Fourier series, Proc. Nat. Acad. Sci. U.S.A. 41 (1955), 558-591. 
[2] Gát G., Almost everywhere convergence of Fejér and logarithmic means of subsequences of partial sums of the Walsh-Fourier series of integrable functions, J. Approx. Theory 162 (2010), 687-708.

[3] Gát G., Karagulyan G., On convergence properties of tensor products of some operator sequences, J. Geom. Anal. (2015), DOI 10.1007/s12220-015-9662-y.

ROMAN GER: On a functional equation connected with the distributivity of fuzzy implications (Joint work with Marcin E. Kuczma, Wanda Niemyska)

The distributivity law for a fuzzy implication $S:[0,1]^{2} \rightarrow[0,1]$ with respect to a fuzzy conjunction $I:[0,1]^{2} \rightarrow[0,1]$ states that a functional equation

$$
I(x, S(y, z))=S(I(x, y), I(x, z))
$$

is satisfied for all pairs $(x, y)$ from the unit square. To solve this equation in a reasonable class of fuzzy implications in question, Wanda Niemyska has reduced the problem to the study of the following two functional equations:

$$
h(\min (x g(y), 1))=\min (h(x)+h(x y), 1), \quad x \in(0,1), y \in(0,1],
$$

and

$$
h(x g(y))=h(x)+h(x y), \quad x, y \in(0, \infty)
$$

in the class of increasing bijections $h:[0,1] \rightarrow[0,1]$ with an increasing function $g:(0,1] \rightarrow[1, \infty)$ and in the class of monotonic bijections $h:(0, \infty) \rightarrow$ $(0, \infty)$ with a function $g:(0, \infty) \rightarrow(0, \infty)$, respectively.

A description of solutions in much more general classes of unknown functions (including nonmeasurable ones) will be discussed and reported on.

\section{AtTILA GiLÁNYi: On conditionally polynomial functions}

In this talk, we investigate conditional polynomial functional equations. We prove that if $Y$ is a linear normed space, $n$ is a nonnegative integer, $A$ and $B$ are real numbers and a function $f: \mathbb{R} \rightarrow Y$ satisfies

$$
\Delta_{y}^{n+1} f(x)=0, \quad x \leq A, y \geq B
$$

then $f$ is a polynomial function of degree $n$, that is, it fulfils

$$
\Delta_{y}^{n+1} f(x)=0, \quad x, y \in \mathbb{R}
$$


TiBor Kiss: On a hierarchy of convexity properties (Joint work with Zsolt Páles)

Let $I \subseteq \mathbb{R}$ be a real interval. During the talk we will need the following definitions.

Definition. We say that $E: I \times I \rightarrow \mathbb{R}$ is a quasideviation if

$-\operatorname{sgn} E(x, u)=\operatorname{sgn}(x-u)$ for all $x, u \in I$,

- the function $u \mapsto E(x, u)$ is continuous for all fixed $x \in I$, and

- the function $u \mapsto-E(y, u) / E(x, u)$ is strictly increasing on the interval ]$x, y[$ for all $x, y \in I$ with $x<y$.

Let $n \geq 2$ be fixed. We define the ( $n$-variable) E-quasideviation mean $N_{E}$ : $I^{n} \rightarrow \mathbb{R}$ so that the equality $\sum_{i=1}^{n} E\left(x_{i}, N_{E}(x)\right)=0$ holds for all $x=$ $\left(x_{1}, \ldots, x_{n}\right) \in I^{n}$.

It can be shown that the function $N_{E}$ exists and it is uniquely determined.

Definition. Let $f: I \rightarrow \mathbb{R}, n \geq 2, N: f(I)^{n} \rightarrow \mathbb{R}$ be a $n$-variable mean and denote $A_{n}: I^{n} \rightarrow \mathbb{R}$ the $n$-variable arithmetic mean on $I$. We say that $f$ is convex with respect to the pair of means $\left(A_{n}, N\right)$, or $f$ is $\left(A_{n}, N\right)$-convex if

$$
f\left(\frac{x_{1}+\cdots+x_{n}}{n}\right) \leq N\left(f\left(x_{1}\right), \ldots, f\left(x_{n}\right)\right), \quad x_{1}, \ldots, x_{n} \in I .
$$

The aim of the talk is to find necessary and sufficient conditions for the implications

(1) $f$ is $\left(A_{n}, N\right)$-convex $\Rightarrow f$ is $\left(A_{m}, M\right)$-convex and

(2) $f$ is $\left(A_{m}, M\right)$-convex $\Rightarrow f$ is $\left(A_{n}, N\right)$-convex,

where $2 \leq n<m$ are arbitrarily fixed and provided that $N: I^{n} \rightarrow \mathbb{R}$ and $M: I^{m} \rightarrow \mathbb{R}$ are $n$ - and $m$-variable, monotone quasideviation means, generated by the same quasideviation, respectively.

\section{REFERENCES}

[1] Páles Zs., Characterization of quasideviation means, Acta. Math. Sci. Hungar. 40 (1982), 456-462.

[2] Páles Zs., General inequalities for quasideviation means, Aequationes Math. 36 (1988), $32-56$.

\section{Katarzyna Kuhlmann: Spaces of real places of function fields}

Spaces of real places play an important role in real algebra and real algebraic geometry. They appear in all settings where non-archimedean orderings have to be studied. We show with an easy example that such settings come up in a very natural way. 
The first systematic study of real places was done by Serge Lang in 1953 . Since then, spaces of real places have been studied by Murray Marshall, Eberhard Becker, Danielle Gondard, Ron Brown and others. But many questions have remained open. In the talk, I present an example of a space of real places with an amazingly rich topological structure. There are plenty of selfsimilarities in this space, which has led us to the question whether some sort of fractality can be defined. These spaces we study are not necessarily metric, and their natural topology is not so easy to handle.

RADOSEAW LUKASIK: Some orthogonality equation with two functions

We describe the solution $(f, g)$ of the equation

$$
\langle f(x) \mid f(y)\rangle=\langle x \mid g(y)\rangle, \quad x, y \in X
$$

where $f: X \rightarrow Y, g: X \rightarrow X, X, Y$ are Hilbert spaces over the same field $\mathbb{K} \in\{\mathbb{R}, \mathbb{C}\}$.

\section{REFERENCES}

[1] Bernau S.J., The square root of positive self-adjoint operator, J. Austral. Math. Soc. 8. (1968), 17-36.

[2] Chmieliński J., Orthogonality equation with two unknown functions, Aequationes Math. 90 (2016), 11-23.

[3] Łukasik R., Wójcik P., Decomposition of two functions in the orthogonality equation, Aequationes Math. 90 (2016), 495-499.

[4] Riesz F., Nagy B.-Sz., Functional analysis, Dover Publications, Inc., New York, 1990.

Judit MAKó: On strongly convex functions (Joint work with Házy Attila)

Our main result gives a connection between strong Jensen convexity and strong convexity type inequalities. We are also looking for the optimal Takagi type function of strong convexity. Finally Hermite-Hadamard type inequalities is examined, too.

Gyula Maksa: The dilogarithm function and the Abel functional equation (Joint work with Zoltán Daróczy)

In the literature, mostly the identities, the applications and the special values of the dilogarithm functions are investigated. In this talk, we deal with the problem of the connection between a famous identity, namely the so-called Abel equation

$$
\left.F(u)+F(v)+F(1-u v)+F\left(\frac{1-u}{1-u v}\right)+F\left(\frac{1-v}{1-u v}\right)=0, \quad u, v \in\right] 0,1[,
$$


and a real version $L i_{2}$ of the dilogarithm function defined by

$$
L i_{2}(x)=\sum_{n=1}^{\infty} \frac{x^{n}}{n^{2}}, \quad x \in[-1,1] .
$$

Fruzsina MÉszÁros: One more multiplicative type functional equation (Joint work with Károly Lajkó)

We investigate the positive and nonnegative solutions of a multiplicative type functional equation stemming from the pexiderized version of an equation, which originally was introduced by T.M.K. Davison.

JanUsz MoraWIEC: Notes on inhomogeneous refinement equations (Joint work with Rafał Kapica)

Assume that $(\Omega, \mathcal{A}, P)$ is a probability space, $K, L: \Omega \rightarrow \mathbb{R}$ are random variables and $g \in L^{1}(\mathbb{R})$. Let $V_{g}$ denote the set of all solutions $f \in L^{1}(\mathbb{R})$ of the inhomogeneous refinement equation

$$
f(x)=\int_{\Omega}|K(\omega)| f(K(\omega) x-L(\omega)) P(d \omega)+g(x) .
$$

It is clear that $V_{g}$ is a linear subspace of $L^{1}(\mathbb{R})$ if and only if $g=0$. Moreover, if $g \neq 0$, then $V_{g}$ is uniquely determined by the subspace $V_{0}$ and by an arbitrary particular solution $f \in L^{1}(\mathbb{R})$ of equation $(1)$, if exists any. During the talk the set $V_{g}$ will be investigated.

\section{NoÉmi NAGY: Approximately Jensen-convex functions}

In this talk we show that if a function satisfies the Jensen-inequality (or the inequality describing $\mathbb{Q}$-convexity) with an appropriate error term then the function is Jensen-convex (without error) as well.

First we consider a function $f$, which is defined on an open interval $I$ of $\mathbb{R}$. Let $\ell_{I}$ be the length of the interval $I$ and define $J_{I}$ as $\left[0, \ell_{I}[\right.$, furthermore let $\psi: J_{I} \rightarrow\left[0,+\infty\left[\right.\right.$ be such that $\lim _{t \rightarrow 0+} \frac{\psi(t)}{t^{2}}=0$. We prove that if $f: I \rightarrow \mathbb{R}$ satisfies the inequality

$$
f\left(\frac{x+y}{2}\right) \leq \frac{f(x)+f(y)}{2}+\psi(|x-y|)
$$

for every $x, y \in I$, then $f$ is Jensen-convex. 
We also prove that if a real function $f$, which is defined on a $\mathbb{F}$-algebraically open $\mathbb{F}$-convex subset $D$ of a vector space $X$ over $\mathbb{F}$ (where $\mathbb{F}$ is a subfield of $\mathbb{R})$, satisfies the inequality

$$
f(\lambda x+(1-\lambda) y) \leq \lambda f(x)+(1-\lambda) f(y)+c[\lambda(1-\lambda)|x-y|]^{p}
$$

for every $x, y \in D$ and $\lambda \in[0,1] \cap \mathbb{F}$, with a fixed non-negative real number $c$ and a fixed exponent $p>1$, then it has to be $\mathbb{F}$-convex, i.e., $f$ satisfies the above inequality with $c=0$ as well. Considering $\mathbb{F}=\mathbb{Q}$, we can see that the last statement can be applied for (approximately) Jensen-convex functions.

\section{ANDRZeJ OlBryś: Inequalities related to Cauchy's functional equation}

We introduce the following definition:

Definition. Let $(S,+)$ be a semigroup. A map $f: S \rightarrow \mathbb{R}$ is said to be quasi-subadditive, if

$$
f(x+y) \leq 2 \max \{f(x), f(y)\}, \quad x, y \in S .
$$

If $f$ is a map such that $-f$ is a quasi-subadditive then we say that it is quasi-superadditive. In this case $f$ satisfies the inequality

$$
2 \min \{f(x), f(y)\} \leq f(x+y), \quad x, y \in S .
$$

If $f$ is at the same time quasi-subadditive and quasi-superadditive then we say that $f$ is quasi-additive.

In the present talk we study the properties of defined mappings.

ZSOLT PÁLES: Comparison of nonsymmetric arithmetic means weighted by weightfunctions

Given $n \geq 2$ and positive functions $f_{1}, \ldots, f_{n}: I \rightarrow \mathbb{R}_{+}$, we can define the $n$-variable arithmetic mean weighted by $\left(f_{1}, \ldots, f_{n}\right)$ denoted by $A_{f_{1}, \ldots, f_{n}}$ : $I^{n} \rightarrow I$ in the following way:

$$
A_{f_{1}, \ldots, f_{n}}\left(x_{1}, \ldots, x_{n}\right):=\frac{f_{1}\left(x_{1}\right) x_{1}+\cdots+f_{n}\left(x_{n}\right) x_{n}}{f_{1}\left(x_{1}\right)+\cdots+f_{n}\left(x_{n}\right)}, \quad x_{1}, \ldots, x_{n} \in I .
$$

Obviously, if $f_{1}=\cdots=f_{n}=1$, then we obtain the standard (symmetric) arithmetic mean. If $f_{1}, \ldots, f_{n}$ are constant functions, then we get the (standard) weighted arithmetic mean.

The aim of the talk is solve the comparison, equality, homogeneity and translativity problem in this class of means. As a consequence of our result, 
it turns out that the comparison and equality of these means and also the homogeneity and translativity are localizable properties.

Bella Popovics: On convexity structures induced by Chebyshev systems (Joint work with Mihály Bessenyei)

Investigating separation problems via generalized affine or convex functions the importance of some results of Convex and Combinatorial Geometry have become clear. For this reason convexity structures induced by Chebyshev systems need to study. It turns out that if the dimension of the Chebyshev system is more then 3 then the results above may radically change. In this talk we determine the so-called Carathéodory-, Radon-, and Helly-numbers of these settings.

Éva SzÉKelyné RAdÁCSI: A new characterization of convexity with respect to Chebyshev systems (Joint work with Zsolt Páles)

The notion of $n$th order convexity is defined via the nonnegativity of the $(n+1)$ st order divided differences of a given real-valued function. In view of the well-known recursive formula for divided differences, the nonnegativity of $(n+1)$ st order divided differences is equivalent to the monotonicity of $n$th order divided differences which provides a characterization of $n$th order convexity.

The aim of this talk is to introduce the notion of higher-order divided differences in the context of convexity with respect to Chebyshev systems. Using a determinantal identity of Sylvester, we then establish a recursive formula of the generalized divided differences of consecutive order which enables us to obtain a new characterization of convexity with respect to Chebyshev systems.

\section{REFERENCES}

[1] Kuczma M., An introduction to the theory of functional equations and inequalities. Cauchy's equation and Jensen's inequality, Birkhäuser Verlag, Basel, 2009.

[2] Páles Zs., Radácsi É.Sz., Characterizations of higher-order convexity properties with respect to Chebyshev systems, Aequationes Math. 90 (2016), 193-210.

PATRICIA SzOKOL: On determinant preserving maps (Joint work with H. Huang, C.-N. Liu, M.-C. Tsai, J. Zhang)

The famous Frobenius theorem on determinant preserving maps can be considered as the first result on linear preserver problems. Motivated by Frobenius' theorem, in this talk we describe the structure of all transformations that preserve the determinant of the sum of two positive definite matrices. It turns out that these maps are closely related to ones that preserve the trace of the 
product of positive definite matrices. We also characterize the map $\phi: S \rightarrow S$ preserving the determinant of convex combinations in $S$, where $S$ can be the set of complex matrices, positive definite matrices, symmetric matrices, and upper triangular matrices.

TOMasz SzostoK: Functional equations and inequalities connected with quadrature rules with weight function

We obtain solutions of the following functional equation

$$
\sum_{i=1}^{k} a_{i} F\left(\alpha_{i} x+\beta_{i} y\right)=(y-x) \sum_{i=1}^{n} b_{i} f\left(\delta_{i} x+\gamma_{i} y\right)
$$

under some assumptions on constants $a_{i}, b_{i}, \alpha_{i}, \beta_{i}, \delta_{i}, \gamma_{i}$. We also present some remarks on inequalities related to (1).

\section{PAWEŁ WóJCIK: On invariant nonlinear subspaces}

Does every operator on a Banach space have an invariant subspace? This is the "invariant subspace problem". The problem which we consider in this report is similar, but not equivalent to the "invariant subspace problem". We discuss the following problem: whether given operator on a Banach space has an invariant nonlinear subspace. We examine the existence or non-existence of such nonlinear subspaces. Moreover, we give some applications.

\section{Problems and Remarks}

1. Remark (A historical remark on the singular case " $p=1$ " of the stability of the Cauchy's functional equation) The following stability problem:

$$
\|f(x+y)-f(x)-f(y)\| \leq c\left(\|x\|^{p}+\|y\|^{p}\right)
$$

has been first discussed by D.H. Hyers in [3] for $p=0$ and then by T. Aoki in [1] for $0 \leq p<1$. Z. Gajda [2] proved the stability for $p \in \mathbb{R} \backslash\{1\}$ and provided a counterexample for $p=1$.

In this remark we want to point out that a counterexample was already known much earlier. Moreover, simple example $f(x)=x \log |x|$ for $x \in \mathbb{R} \backslash$ $\{0\}$ and $f(0)=0$, which is frequently attributed to P. Găvruţa, was firstly observed by M. Ribe [7, Lemma 2]. 
In our opinion the following result of N.J. Kalton [4, basically: Proposition 3.3, Lemma 4.5 and Theorem 4.6] from 1978 should be acknowledged as the historically earliest known counterexample for the stability problem (1) for $p=1$.

Theorem (N.J. Kalton, 1978). Let $\left(e_{n}\right)$ be the usual basis of $\ell^{1}$ and let $E=\operatorname{lin}\left\{e_{1}, e_{2}, \ldots\right\}$. There exists a function $f: E \rightarrow \mathbb{R}$ such that:

(i) $\|f(x+y)-f(x)-f(y)\| \leq(4 \log 2)(\|x\|+\|y\|)$ for all $x, y \in E$,

(ii) $f(s x)=s f(x)$ for all $s \in \mathbb{R}$ and $x \in E$,

(iii) there is no linear function $h: E \rightarrow \mathbb{R}$ and no constant $B \in \mathbb{R}$ such that $\|f(x)-h(x)\| \leq B\|x\|$ for all $x \in E$.

The topic has been developed further and mappings which satisfy (i) (ii) and (iii) of the above statement were studied deeper. Other fundamental results on the same topic are due to N.J. Kalton [5], N.J. Kalton and N.T. Peck [6], J.W. Roberts [8]. It seems that three similar examples were found independently by N.J. Kalton, M. Ribe and J.W. Roberts.

\section{REFERENCES}

[1] Aoki T., On the stability of the linear transformation in Banach spaces, J. Math. Soc. Japan 2 (1980), 64-66.

[2] Gajda Z., On stability of additive mappings, Internat. J. Math. Math. Sci. 14 (1991), 431-434.

[3] Hyers D.H., On the stability of the linear functional equation, Proc. Nat. Acad. Sci. U.S.A. 27 (1941), 222-224.

[4] Kalton N.J., The three space problem for locally bounded F-spaces, Compositio Math. 37 (1978), 243-276.

[5] Kalton N.J., Convexity, type and the three space problem, Studia Math. 69 (1980/81), $247-287$.

[6] Kalton N.J., Peck N.T., Twisted sums of sequence spaces and the three space problem, Trans. Amer. Math. Soc. 255 (1979), 1-30.

[7] Ribe M., Examples for the nonlocally convex three space problem, Proc. Amer. Math. Soc. 73 (1979), 351-355.

[8] Roberts J.W., A nonlocally convex F-space with the Hahn-Banach approximation property, in: Banach spaces of analytic functions (Proc. Pelczynski Conf., Kent State Univ., Kent, Ohio, 1976), Lecture Notes in Math., Vol. 604, Springer, Berlin, 1977, pp. 76-81.

\section{WŁODZIMIERZ FeChNeR}

2. REMARK (On the essential union and intersection of families of measurable sets) The notions that we discuss below turn out to be significant if one tries to extend the Radon-Nikodym Theorem for non- $\sigma$-finite measure spaces. For details, we refer to Section 1.1 and pages $65-71$ of the recent monograph [1] by Fonseca and Leoni. More comments will be given at the end of this note. 
Given a measure space $(X, \mathcal{A}, \mu)$, we say that a set $A \in \mathcal{A}$ is $\mu$-contained in $B \in \mathcal{A}$ if $\mu(A \backslash B)=0$ and we denote this fact by $A \subseteq_{\mu} B$. Analogously, a set $A \in \mathcal{A}$ is said to be $\mu$-equal to $B \in \mathcal{A}$ if $\mu((A \backslash B) \cup(B \backslash A))=0$ holds and this property is denoted as $A={ }_{\mu} B$. It is easy to see that $=_{\mu}$ is an equivalence relation on the $\sigma$-algebra $\mathcal{A}$, and $\subseteq_{\mu}$ is a partial ordering on the equivalence classes. It can also be shown that the function $d_{\mu}$ defined by

$$
d_{\mu}(A, B):=\mu((A \backslash B) \cup(B \backslash A)), \quad A, B \in \mathcal{A},
$$

is a semimetric on $\mathcal{A}$ and is a metric on the equivalence classes of the relation $={ }_{\mu}$.

For a family $\left\{A_{\gamma}: \gamma \in \Gamma\right\} \subseteq \mathcal{A}$ of measurable subsets, we say that a set $\bar{A} \in \mathcal{A}$ is its $\mu$-essential union if

$$
A_{\gamma} \subseteq_{\mu} \bar{A}, \quad \gamma \in \Gamma
$$

and if, for $A^{*} \in \mathcal{A}$,

$$
A_{\gamma} \subseteq_{\mu} A^{*}, \quad \gamma \in \Gamma,
$$

holds, then $\bar{A} \subseteq_{\mu} A^{*}$. The notion of the $\mu$-essential intersection is defined completely in a similar manner: A set $\underline{A} \in \mathcal{A}$ is called the $\mu$-essential intersection of the family $\left\{A_{\gamma}: \gamma \in \Gamma\right\} \subseteq \mathcal{A}$ if

$$
\underline{A} \subseteq_{\mu} A_{\gamma}, \quad \gamma \in \Gamma
$$

and if, for $A_{*} \in \mathcal{A}$,

$$
A_{*} \subseteq_{\mu} A_{\gamma}, \quad \gamma \in \Gamma,
$$

is satisfied, then $A_{*} \subseteq \underline{\underline{A}} \underline{\underline{A}}$.

Clearly, if the sets $\bar{A}, \underline{A} \in \mathcal{A}$ exist, then they are uniquely determined up to the equivalence $=_{\mu}$. It is also easy to see that, for all countable subset $\Gamma_{0} \subseteq \Gamma$,

$$
\underline{A} \subseteq_{\mu} \bigcap_{\gamma \in \Gamma_{0}} A_{\gamma}, \quad \bigcup_{\gamma \in \Gamma_{0}} A_{\gamma} \subseteq_{\mu} \bar{A}
$$

are valid. On the other hand, in general, it is not true that $\underline{A} \subseteq_{\mu} \bigcap_{\gamma \in \Gamma} A_{\gamma}$, and $\bigcup_{\gamma \in \Gamma} A_{\gamma} \subseteq_{\mu} \bar{A}$. To see the clear difference from the standard notions of union and intersection, consider the following example. Let $(\mathbb{R}, \mathcal{L}, \lambda)$ be the standard Lebesgue measure space on $\mathbb{R}$. Then, for any non-Lebesgue measurable subset $\Gamma \subseteq \mathbb{R}$, the essential union of the family $\{\{\gamma\}: \gamma \in \Gamma\}$ is the empty set because $\lambda(\{\gamma\} \backslash \emptyset)=0$ for all $\gamma \in \Gamma$. On the other hand, the standard union of this family is $\Gamma$, which cannot be $\lambda$-equal to the empty set because it is non-measurable. 
The following theorem establishes the existence of the essential union and intersection of arbitrary families of measurable sets in $\sigma$-finite measure spaces.

Theorem. Let $(X, \mathcal{A}, \mu)$ be a $\sigma$-finite measure space. Then any family $\left\{A_{\gamma}: \gamma \in \Gamma\right\} \subseteq \mathcal{A}$ of measurable sets has a $\mu$-essential union and a $\mu$-essential intersection.

For the proof of the above Theorem, we shall need the following auxiliary result.

Lemma. If $(X, \mathcal{A}, \mu)$ is a $\sigma$-finite measure space then there exists a finite measure $\nu$ on $\mathcal{A}$ such that $\mu$ and $\nu$ are mutually absolutely continuous with respect to each other, that is, $\mu(A)=0$ holds if and only if $\nu(A)=0$.

Proof. The statement is trivial if $\mu(X)<\infty$, therefore we may assume that $\mu(X)=\infty$. Let $A_{1}, A_{2}, \ldots$ be pairwise disjoint measurable sets such that $X=\bigcup_{n=1}^{\infty} A_{n}$ and $0<\mu\left(A_{n}\right)<\infty$ for all $n \in \mathbb{N}$. Then define $\nu$ in the following way:

$$
\nu(A):=\sum_{n=1}^{\infty} \frac{\mu\left(A \cap A_{n}\right)}{2^{n} \mu\left(A_{n}\right)}, \quad A \in \mathcal{A} .
$$

One can easily verify that $\nu$ is a measure on $\mathcal{A}, \nu(X)=1$, and $\mu$ and $\nu$ are mutually absolutely continuous with respect to each other.

Proof of The Theorem. It is sufficient to prove the statement about the existence of the $\mu$-essential union, because the assertion for the $\mu$-essential intersection follows from this by switching to complements of the sets belonging to the given family. In view of the Lemma, we can assume that $\mu(X)<\infty$. We may also assume that the family $\left\{A_{\gamma}: \gamma \in \Gamma\right\}$ is closed under countable union.

We are going to show that, for all $n \in \mathbb{N}$, there exists $\gamma_{n} \in \Gamma$ such that

$$
\mu\left(A_{\gamma} \backslash A_{\gamma_{n}}\right)<\frac{1}{n}, \quad \gamma \in \Gamma .
$$

Let $\gamma_{n_{1}} \in \Gamma$ be arbitrary. If, for all $\gamma \in \Gamma$, we have $\mu\left(A_{\gamma} \backslash A_{\gamma_{n_{1}}}\right)<\frac{1}{n}$, then the choice $\gamma_{n}:=\gamma_{n_{1}}$ completes the argument. In the other case, there exists $\gamma^{\prime} \in \Gamma$ such that $\mu\left(A_{\gamma^{\prime}} \backslash A_{\gamma_{n_{1}}}\right) \geq \frac{1}{n}$. Since, the family is closed under intersection, there exists $\gamma_{n_{2}} \in \Gamma$ such that $A_{\gamma^{\prime}} \cup A_{\gamma_{n_{1}}}=A_{\gamma_{n_{2}}}$. Then

$$
A_{\gamma_{n_{2}}} \supseteq A_{\gamma_{n_{1}}} \quad \text { and } \quad \mu\left(A_{\gamma_{n_{2}}}\right) \geq \mu\left(A_{\gamma_{n_{1}}}\right)+\frac{1}{n} .
$$


If, for all $\gamma \in \Gamma$, we have $\mu\left(A_{\gamma} \backslash A_{\gamma_{n_{2}}}\right)<\frac{1}{n}$, then with $\gamma_{n}:=\gamma_{n_{2}}$ we are done. Otherwise, there exists $\gamma^{\prime} \in \Gamma$ such that $\mu\left(A_{\gamma^{\prime}} \backslash A_{\gamma_{n_{2}}}\right) \geq \frac{1}{n}$. Then there exists $\gamma_{n_{3}} \in \Gamma$ such that $A_{\gamma^{\prime}} \cup A_{\gamma_{n_{2}}}=A_{\gamma_{n_{3}}}$ and we have

$$
A_{\gamma_{n_{3}}} \supseteq A_{\gamma_{n_{2}}} \quad \text { and } \quad \mu\left(A_{\gamma_{n_{3}}}\right) \geq \mu\left(A_{\gamma_{n_{2}}}\right)+\frac{1}{n} .
$$

Continuing this procedure, it terminates after finitely many steps, because the measure space is finite and the measure of the sequence increases by $\frac{1}{n}$ in each step.

Finally, let

$$
\bar{A}:=\bigcup_{n=1}^{\infty} A_{\gamma_{n}} .
$$

Then, for all $\gamma \in \Gamma$,

$$
\mu\left(A_{\gamma} \backslash \bar{A}\right)<\frac{1}{n}, \quad n \in \mathbb{N},
$$

therefore $\mu\left(A_{\gamma} \backslash \bar{A}\right)=0$, i.e., $A_{\gamma} \subseteq_{\mu} \bar{A}$.

If $A^{*} \in \mathcal{A}$ has similar properties as $\bar{A}$, then it $\mu$-contains $A_{\gamma_{n}}$ for all $n \in \mathbb{N}$, hence it should $\mu$-contain $\bar{A}$, too.

The $\sigma$-finiteness of the measure space is neither necessary nor superfluous for the conclusion of the theorem. To see this, let $\mathcal{A}$ be the $\sigma$-algebra of all countable and co-countable subsets of $\mathbb{R}$ and define two measures, $\mu$ and $\nu$ as follows: let $\mu(A):=0$ if $A$ is countable and $\mu(A):=\infty$ if $A$ is co-countable (i.e., its complement is countable), and let $\nu$ be the counting measure, i.e., let $\nu(A):=n$ if $A$ is finite and contains $n$ elements and let $\nu(A):=\infty$ if $A$ has infinitely many elements. Obviously, the two measure spaces $(X, \mathcal{A}, \mu)$ and $(X, \mathcal{A}, \nu)$ are not $\sigma$-finite.

First we show that the conclusion of the Theorem holds in $(X, \mathcal{A}, \mu)$. Let $\left\{A_{\gamma}: \gamma \in \Gamma\right\} \subseteq \mathcal{A}$ be a family of measurable sets. If, for each $\gamma \in \Gamma$, the set $A_{\gamma}$ is countable, then the empty set (moreover, any countable set) can be chosen as the $\mu$-essential union of the family. If, for some $\gamma_{0} \in \Gamma, A_{\gamma_{0}}$ is co-countable, then $\mathbb{R}$ (or any co-countable set) can be chosen as the $\mu$-essential union of the family.

Finally, let $\Gamma \subseteq \mathbb{R}$ be a non-countable set such that its complement is also not countable. We show that the family $\{\{\gamma\}: \gamma \in \Gamma\}$ cannot have a $\nu$-essential union. To see this, observe that a set $A \in \mathcal{A}$ has $\nu$ measure zero if and only if $A=\emptyset$. Thus, $A \subseteq_{\nu} B$ is equivalent to the standard inclusion $A \subseteq B$. Hence, the $\nu$-essential union of the family $\{\{\gamma\}: \gamma \in \Gamma\}$ should be 
equal to the set $\Gamma$, which does not belong to $\mathcal{A}$, i.e., which is not measurable (this second example was also observed by Zoltán Boros during this meeting).

The following result was established in [1, Theorem 108].

Corollary. Let $(X, \mathcal{A}, \mu)$ be a $\sigma$-finite measure space. Then, for any family $\left\{f_{\gamma}: X \rightarrow[-\infty,+\infty] \mid \gamma \in \Gamma\right\}$ of extended real-valued measurable functions, there exist measurable functions $f, \bar{f}: X \rightarrow[-\infty,+\infty]$ such that

$$
\underline{f} \leq_{\mu} f_{\gamma} \leq_{\mu} \bar{f}, \quad \gamma \in \Gamma,
$$

and if $f_{*}, f^{*}: X \rightarrow[-\infty,+\infty]$ are measurable functions such that

$$
f_{*} \leq_{\mu} f_{\gamma} \leq_{\mu} f^{*}, \quad \gamma \in \Gamma,
$$

then $f_{*} \leq_{\mu} \underline{f}$ and $\bar{f} \leq_{\mu} f^{*}$.

The $\mu$-uniquely determined functions $f$ and $\bar{f}$ defined in the above corollary are called the essential infimum and essential supremum of the family of measurable functions (cf. [1, Definition 106]). It is easy to see that, for all countable subset $\Gamma_{0} \subseteq \Gamma$,

$$
\underline{f} \leq_{\mu} \inf _{\gamma \in \Gamma_{0}} f_{\gamma}, \quad \sup _{\gamma \in \Gamma_{0}} f_{\gamma} \leq_{\mu} \bar{f} .
$$

On he other hand, in general, it is not true that $\underline{f} \leq_{\mu} \inf _{\gamma \in \Gamma} f_{\gamma}$, and that $\sup _{\gamma \in \Gamma} f_{\gamma} \leq_{\mu} \bar{f}$.

The above result can easily be deduced from the Theorem by applying it to the epigraphs of the functions and observing that, for two extended realvalued functions $f, g: X \rightarrow[-\infty,+\infty]$ the relation $f \leq_{\mu} g$ (i.e., the inequality $f \leq g$ holds $\mu$ almost everywhere) if and only if epi $g \subseteq_{\mu \otimes \lambda}$ epi $f$, where $\mu \otimes \lambda$ denotes the product measure of $\mu$ and the Lebesgue measure $\lambda$.

In the book [1] first the notions of $\mu$-essential supremum and infimum of families of measurable functions is defined. The concepts of $\mu$-essential union and intersection of families of measurable sets is then introduced via the $\mu$ essential supremum and infimum of their characteristic functions. In this note we followed a different approach, we defined the notions $\mu$-essential union and intersection of families of measurable sets first.

Those measure spaces where the conclusion of our Theorem remains valid are called localizable by the terminology of the book [1]. It turns out that the Radon-Nikodym Theorem remains valid for measures on such spaces. 


\section{REFERENCE}

[1] Fonseca I., Leoni G., Modern methods in the calculus of variations: $L^{p}$ spaces. Springer Monographs in Mathematics, Springer Verlag, Berlin, 2007.

Zsolt PÁles

\section{List of Participants}

Roman Badora, Institute of Mathematics, University of Silesia, ul. Bankowa 14, 40-007 Katowice, Poland; e-mail: roman.badora@us.edu.pl

Zoltán Boros, Institute of Mathematics, University of Debrecen, Pf. 400, 4002 Debrecen, Hungary; e-mail: zboros@science.unideb.hu

PÁl Burai, Faculty of Informatics, University of Debrecen, Pf. 400, 4002 Debrecen, Hungary; e-mail: burai.pal@inf.unideb.hu

Zoltán Daróczy, Institute of Mathematics, University of Debrecen, Pf. 400, 4002 Debrecen, Hungary; e-mail: daroczy@science.unideb.hu

Szymon Draga, Institute of Mathematics, University of Silesia, ul. Bankowa 14, 40-007 Katowice, Poland; e-mail: szymon.draga@gmail.com

WŁodzimierz Fechner, Institute of Mathematics, University of Silesia, ul. Bankowa 14, 40-007 Katowice, Poland; e-mail: fechner@math.us.edu.pl

GYÖRGY GÁT, Institute of Mathematics, University of Debrecen, Pf. 400, 4002 Debrecen, Hungary; e-mail: gat.gyorgy@science.unideb.hu

Roman Ger, Institute of Mathematics, University of Silesia, ul. Bankowa 14, 40-007 Katowice, Poland; e-mail: romanger@us.edu.pl

Attila Gilányi, Faculty of Informatics, University of Debrecen, Pf. 400, 4002 Debrecen, Hungary; e-mail: gilanyi.attila@inf.unideb.hu

TiBor Kiss, Institute of Mathematics, University of Debrecen, Pf. 400, 4002 Debrecen, Hungary; e-mail: kiss.tibor@science.unideb.hu

ZyGfryd KomineK, Institute of Mathematics, University of Silesia, ul. Bankowa 14, 40-007 Katowice, Poland; e-mail: zkominek@math.us.edu.pl

Katarzyna Kuhlmann, Institute of Mathematics, University of Silesia, ul. Bankowa 14, 40-007 Katowice, Poland; e-mail: kmk@math.us.edu.pl 
RadosŁaW Łukasik, Institute of Mathematics, University of Silesia, ul. Bankowa 14, 40-007 Katowice, Poland; e-mail: rlukasik@math.us.edu.pl

Judit Makó, Department of Analysis, University of Miskolc, 3515 MiskolcEgyetemváros, Hungary; e-mail: matjudit@uni-miskolc.hu

Gyula Maksa, Institute of Mathematics, University of Debrecen, Pf. 400, 4002 Debrecen, Hungary; e-mail: maksa@science.unideb.hu

Fruzsina MÉszÁros, Institute of Mathematics, University of Debrecen, Pf. 400, 4002 Debrecen, Hungary; e-mail: mefru@science.unideb.hu

Janusz Morawiec, Institute of Mathematics, University of Silesia, ul. Bankowa 14, 40-007 Katowice, Poland; e-mail: janusz.morawiec@us.edu.pl

NoÉmi Nagy, Department of Applied Mathematics, University of Miskolc, 3515 Miskolc-Egyetemváros, Hungary; e-mail: matnagyn@uni-miskolc.hu

Andrzej Olbryś, Institute of Mathematics, University of Silesia, ul. Bankowa 14, 40-007 Katowice, Poland; e-mail: andrzej.olbrys@wp.pl

Zsolt PÁles, Institute of Mathematics, University of Debrecen, Pf. 400, 4002 Debrecen, Hungary; e-mail: pales@science.unideb.hu

Bella Popovics, Institute of Mathematics, University of Debrecen, Pf. 400, 4002 Debrecen, Hungary; e-mail: popovics.bella@science.unideb.hu

Maciej Sablik, Institute of Mathematics,University of Silesia, ul. Bankowa 14, 40-007 Katowice, Poland; e-mail: maciej.sablik@us.edu.pl

Justyna Sikorska, Institute of Mathematics, University of Silesia, ul. Bankowa 14, 40-007 Katowice, Poland; e-mail: justyna.sikorska@us.edu.pl

Éva SzÉKelyné Radécsi, Institute of Mathematics, University of Debrecen, Pf. 400, 4002 Debrecen, Hungary; e-mail: radacsi.eva@science.unideb.hu

PatríCia Szokol, Department of Analysis, University of Miskolc, 3515 MiskolcEgyetemváros, Hungary; e-mail: szokolp@science.unideb.hu

Tomasz Szostok, Institute of Mathematics, University of Silesia, ul. Bankowa 14, 40-007 Katowice, Poland; e-mail: tszostok@math.us.edu.pl

PaWę WóJcik, Institute of Mathematics, Pedagogical University of Cracow, Podchorążych 2, 30-084 Kraków; Poland; e-mail: pwojcik@up.krakow.pl 